\title{
RFID Technologies: Ausgewählte Netzwerk-, Weiterbildungs- und Forschungsaktivitäten in Österreich
}

\author{
J. Grosinger, W. Bösch
}

Online publiziert am 25. Mai 2016

(c) Springer Verlag Wien 2016

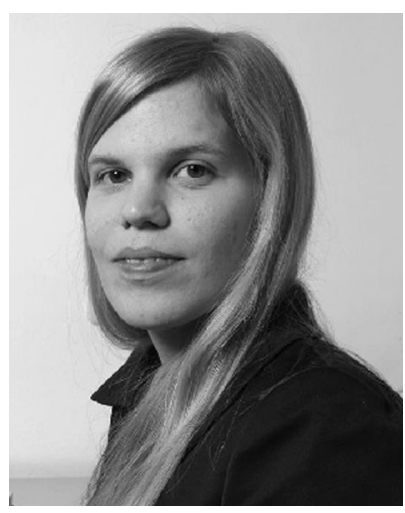

Dipl.-Ing. Dr.

Jasmin Grosinger, B.Sc.

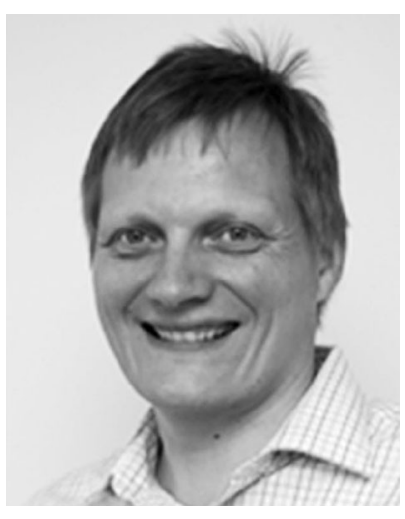

Univ.-Prof. Dipl.-Ing. Dr. Wolfgang Bösch, MBA
Die Forschung und Entwicklung von Radio Frequency Identification (RFID) Technologies ist in Österreich allgegenwärtig. Vor allem deshalb, weil die RFID Technologies zu den Schlüsseltechnologien zählen, um die Vision des Internet der Dinge zu realisieren. Im Bereich der Forschung und Entwicklung von RFID Technologies stehen vor allem der Einsatz im täglichen Leben, aber auch Anwendungen aus der Industrie im Fokus.

Ein Forschungs- und Entwicklungsschwerpunkt im Bereich der RFID Technologies liegt auf der Seite der Hochfrequenztechnik. Hier arbeiten vor allem auch die Mitglieder der Arbeitsgemeinschaft Hochfrequenztechnik (ARGE HFT) der Österreichischen Forschungsgemeinschaft gemeinsam mit Partnern aus der Industrie in kooperativen Forschungsprojekten an Innovationen im Bereich RFID Technologies. Die ARGE HFT (http://

www.oefg.at/de/arbeitsgemeinschaften/hochfrequenztechnik/) wurde 2013 gegründet und setzt sich nicht nur aus den drei traditionellen Hochfrequenztechnik-Instituten an den österreichischen Technischen Universitäten zusammen, sondern umfasst interdisziplinär auch Institute, die sich mit der digitalen Signalverarbeitung auseinandersetzen. Insgesamt sind derzeit fünf Institute in der Arbeitsgemeinschaft tätig: das Institut für Hochfrequenztechnik sowie das Signal Processing and Speech Communication Laboratory an der Technischen Universität in Graz, das Institute of Telecommunications sowie das Institute of Electrodynamics, Microwave and Circuit Engineering an der Technischen Universität in Wien und das Institute for Communications Engineering and RF-Systems an der Johannes Kepler Universität Linz. Ein Handbuch zur ARGE HFT und deren Forschungsaktivitäten im Bereich RFID Technologies kann über die Österreichische Forschungsgemeinschaft (ÖFG) bezogen werden.
Ein lokaler Schwerpunkt im Bereich RFID Technologies liegt in der Steiermark. Die Entwicklung von RFID Technologies hat in der Steiermark eine lange Tradition. Steirische RFID-Firmen haben in der Branche ein hohes Renommee: Immerhin wurden mehr als $50 \%$ der weltweit im Einsatz befindlichen Chips in der Steiermark entwickelt. Fast alle Firmen sind international und weltweit tätig und beschäftigen im Grazer Raum an die 2.000 Mitarbeiter/innen. Der Exportanteil der steirischen Branche liegt bei über $90 \%$. Aufgrund dieses Schwerpunktes wurde von der Steirischen Wirtschaftsförderungsgesellschaft SFG die Hotspot-Initiative "RFID-Hotspot Steiermark" ins Leben gerufen. Manfred Hall von der SFG stellt im Artikel "RFIDHotspot Steiermark" diese Netzwerkaktivität vor.

Im Weiteren werden in Österreich auch Weiterbildungsaktivitäten im Bereich RFID Technologies verfolgt. Das Institut Life Long Learning der Technischen Universität Graz hat im Zuge des Förderprogramms "Forschungskompetenzen für die Wirtschaft" der Österreichischen Forschungsförderungsgesellschaft FFG RFID-Qualifizierungsnetze ins Leben gerufen, besonders um regionalen Wissenstransfer an Unternehmen zu ermöglichen und zu stärken. Christine Stöckler-Penz, Leiterin des TU Graz Life Long Learning Institutes, stellt im Artikel „RFID-Qualifizierungsnetzwerke: Forschungs-Know-how für Unternehmen" diese Weiterbildungsaktivitäten vor.

Die in diesem Themenheft publizierten Originalarbeiten stellen einen Ausschnitt der Forschungsaktivitäten im Bereich RFID Technologies dar. In den letzten drei Jahren wurde im Zuge des COMETProgramms des Bundes (Competence Centers for Excellent Technologies) das K-Projekt SeCoS - Secure Contactless Sphere: Smart RFID Technologies for a Connected World (http://comet-secos.at/, siehe auch e\&i-Heft 7.2013) durchgeführt. SeCoS hatte zum Ziel, mittels Einsatz von smarten RFID-Technologien eine vernetzte Welt zu schaffen. In einer solchen Welt sind Gegenstände des alltäglichen Lebens untereinander und mit dem Internet vernetzt, um damit unser Leben sicherer und komfortabler zu gestalten und die Effizienz in Betriebsstätten im Bereich der Logistik zu erhöhen. Unter der Führung von Joanneum Research in Graz vereinte SeCoS international führende wissenschaftliche Gruppen und weltweit agierende Unternehmenspartner aus der gesamten RFID-Wertschöpfungskette. Das K-Projekt SeCoS umfasste drei Subprojekte "Web of Things Application Platform", "Integrated Secure Technologies" und "Contactless Transmission Technologies", deren Forschungs- und Entwicklungsergebnisse in fünf "Future Application Demonstrators" zusammenflossen. Die publizierten Originalarbeiten geben Einblick in die Forschungsund Entwicklungsergebnisse von SeCoS.

Grosinger, Jasmin, Institut für Hochfrequenztechnik, Technische Universität Graz, Inffeldgasse 12, 8010 Graz, Österreich (E-Mail: jasmin.grosinger@tugraz.at); Bösch, Wolfgang, Institut für Hochfrequenztechnik, Technische Universität Graz, Inffeldgasse 12, 8010 Graz, Österreich (E-Mail: wbosch@tugraz.at) 
Herwig Zeiner et al. zeigen im Artikel "SeCoS: Web of Things Platform Based on a Microservices Architecture and Support of Time-Awareness" eine intelligente Verknüpfung von realen Objekten mit Softwarediensten für die Realisierung von neuen RFIDAnwendungen.

Lukas Zöscher et al. präsentieren im Artikel „HF/UHF Dual Band RFID Transponders for an Information-Driven Public Transportation System" die Entwicklung eines Dual Band RFID Transponders in der 40-nm-CMOS-Technologie für ein elektronisches Zahlungssystem in einem informationszentrierten, öffentlichen Verkehrssystem, der den Schutz der Privatsphäre von Passagieren garantiert.

Philipp Freidl et al. zeigen die Entwicklung und Realisierung eines miniaturisierten RFID-Systems im Millimeterwellen(mm)-Frequenzbereich im Artikel „mm-Wellen RFID: Design und Implementierung eines Demonstrators".
Lukas Görtschacher et al. präsentieren im Artikel „SIMO UHF RFID Reader Using Sensor Fusion for Tag Localization in a Selected Environment" den Entwurf und den Aufbau eines RFID-Lesegeräts im Ultra High Frequency(UHF)-Frequenzbereich zur Auffindung von mit RFID-Transponder versehenen Messgeräten in einem Motorprüfstand.

Die Artikel in dieser e\&i-Ausgabe geben einen kleinen Einblick auf die vielfältigen und sehr beeindruckenden Netzwerk-, Weiterbildungs- und Forschungsaktivitäten im Bereich RFID Technologies in Österreich. Wir möchten uns an dieser Stelle sehr herzlich bei den Autoren der Beiträge bedanken und wünschen viel Vergnügen beim Lesen dieser Ausgabe. 